Annuaire suisse de politique de développement

$14 \mid 1995$

Annuaire Suisse - Tiers Monde 1995

\title{
VI. Coopération au développement et aide humanitaire
}

\section{OpenEdition}

Journals

Édition électronique

URL : http://journals.openedition.org/aspd/1467

DOI : 10.4000/aspd.1467

ISSN : 1663-9669

Éditeur

Institut de hautes études internationales et du développement

\section{Édition imprimée}

Date de publication : 1 février 1995

Pagination : 121-140

ISSN : 1660-5934

Référence électronique

«VI. Coopération au développement et aide humanitaire », Annuaire suisse de politique de

développement [En ligne], 14 | 1995, mis en ligne le 04 mai 2013, consulté le 08 septembre 2020. URL: http://journals.openedition.org/aspd/1467 ; DOI : https://doi.org/10.4000/aspd. 1467 


\section{Coopération au développement et aide humanitaire ${ }^{(1)}$}

Résumé

Au cours de la période considérée, la coopération publique et privée au développement de la Suisse a été confrontée à une foule de nouvelles tâches dans les pays en développement et dans les pays en transition, tout en subissant des pressions budgétaires croissantes en Suisse. Outre les instruments traditionnels que sont la coopération technique, l'aide financière, l'aide humanitaire et les mesures de politique économique et commerciale, le changement politique et social, parallèlement à l'évolution structurelle de l'économie mondiale, posent de nouvelles exigences à la coopération au développement. Puisque tout le monde s'accorde désormais pour reconnaître l'interdépendance des relations entre pays pauvres et pays riches, ainsi que le fait que seules des actions cohérentes orientées sur le long terme peuvent conduire à un développement durable pour tous, les acteurs de la coopération au développement doivent non seulement poursuivre les projets classiques, mais en lancer de nouveaux dans le domaine de la politique de développement (encourager le processus de démocratisation, promouvoir la paix, créer de nouvelles institutions, lutter pour la préservation de l'environnement, etc.). Dans le même temps, l'augmentation du déficit des finances fédérales a nettement accru les pressions budgétaires. Le Parlement a ainsi réduit le crédit de programme pour la coopération technique et l'aide financière. Les crédits de paiement destinés à la coopération au développement que le Parlement doit 
approuver chaque année ont aussi subi des coupes. En 1993, le volume des dons destinés aux oeuvres privées d'entraide a également diminué par rapport à l'année précédente. Cette évolution oblige les responsables privés et publics de la coopération au développement d'unir et de mieux cibler leurs efforts. II s'agit concrètement de définir des priorités: répartir l'aide bilatérale entre un nombre moins grand de pays et de projets, ainsi que diminuer les dépenses pour l'aide multilatérale. Ces efforts qui visent une meilleure efficacité de l'aide alors que les moyens se font plus rares doivent s'orienter sur le long terme. La Direction de la coopération au développement et de l'aide humanitaire (DDA) a fait part de son intention de réduire encore dans les années à venir le nombre des pays de concentration de l'aide, qui sont aujourd'hui au nombre de 18 .

Pendant la période en revue (1993 à septembre 1994), la Confédération a publié d'importants documents qui constituent les fondements de la politique suisse de développement pour les années nonante. II s'agit du Rapport sur la politique extérieure de la Suisse dans les années 90, du Rapport du Conseil fédéral sur les relations Nord-Sud de la Suisse dans les années 90 ("Lignes directrices Nord-Sud") et du Message concernant la continuation de la coopération technique et de l'aide financière en faveur des pays en développement.

Des expériences réalisées lors de la conférence des Nations Unies sur l'environnement et le développement à Rio en 1992 ainsi qu'à la Conférence sur la population au Caire en 1994 et des préparatifs précédant le Sommet sur le développement social et la Conférence sur les femmes (tous deux prévus pour 1995), on peut tirer une seule et même conclusion: les problèmes de développement ne peuvent être considérés et résolus que dans un contexte global. C'est aussi l'avis du Conseil fédéral lorsqu'il écrit dans son introduction au rapport sur la politique extérieure mentionné ci-dessus (p. 5): "En effet, ce n'est plus l'inertie de l'ordre mondial bipolaire de l'après-guerre, mais bien la dynamique de la "politique intérieure mondiale" qui constitue aujourd'hui le cadre de l'élaboration et de l'examen de notre politique extérieure." Dans un monde fait d'interdépendances, la politique suisse de développement devrait s'intégrer dans une politique extérieure aussi cohérente que possible. Les "Lignes directrices Nord-Sud" et le Rapport sur la politique extérieure de la Suisse dans les années 90 s'attachent donc principalement à formuler une politique suisse aussi cohérente que possible dans ses relations extérieures. Ce sujet est le thème principal de la deuxième partie du présent annuaire: Cohérence des politiques dans les relations de la Suisse avec I'Indonésie (2).

En juin 1994, le peuple suisse a été invité à se rendre aux urnes pour donner son avis sur la participation de la Suisse au contingent des casques bleus de l'ONU qui assurent les opérations de maintien de la paix. Une faible majorité populaire s'est dessinée en faveur de cette mesure, tandis que la majorité des cantons s'y est opposée, ce qui a fait échouer le projet. 


\section{Aide publique au développement}

\section{Rapport annuel DDA / OFAEE}

Le rapport annuel commun de la DDA et de l'OFAEE aborde en 1993 l'encouragement du secteur privé, le désendettement et la question démographique et donne quelques exemples de mesures bilatérales et multilatérales prises par la Confédération dans ces domaines. La Suisse s'est engagée avec beaucoup de détermination pour la promotion du secteur privé qui constitue selon elle un moteur important du développement autonome d'un pays. A son avis, les modèles de développement ont négligé pendant longtemps le rôle du secteur privé: "Naguère encore, les stratégies de développement attribuaient à l'Etat une fonction toute-puissante. En l'absence d'autres structures influentes, il ne devait pas se contenter d'être un guide: il devait être le principal agent économique et financier du pays." (Rapport annuel 1993, p. 6) La Suisse soutient principalement la promotion des petites et moyennes entreprises et de l'artisanat giâce à la coopération technique. Une nouvelle priorité réside dans l'encouragement d'organisations, d'institutions et d'associations qui renforcent les structures des activités du secteur privé; c'est par exemple le cas au Viêt-nam. La Suisse a conclu avec le Viêt-nam une série d'accords concernant la coopération économique et financière. En 1993, elle a participé à raison de 23,3 millions de francs au développement et à la reconstruction du pays, ce qui place le Viêt-nam en troisième position parmi les pays en développement asiatiques, derrière l'Inde $(39,7$ millions de francs) et l'Indonésie $(38,4)$. Dès 1995 , le Viêtnam comptera parmi les principaux bénéficiaires de l'aide suisse. Quant à I'Indonésie, la DDA compte s'en retirer dès 1996, car son analyse indique que les instruments de l'Office fédéral des affaires économiques extérieures (OFAEE) représentent la forme d'aide au développement qui convient le mieux à la situation dans ce pays.

La Conférence annuelle de la DDA 1994 a eu pour thème la mise en pratique de l'Agenda 21 et le suivi de Rio dans la coopération suisse au développement.

\section{Développement équilibré hommes-femmes}

L'idée que l'on se fait à la DDA d'un développement équitable pour les femmes et les hommes repose sur la notion de "gender balanced development", le "développement équilibré hommes-femmes". Cette nouvelle notion remplace ainsi celle de "promotion des femmes" qui prévalait jusqu'alors. En février 1994, la DDA a publié ses lignes directrices pour les années 90 dans ce domaine sous le titre "Programme transversal". Une remise en question des idées devrait parvenir à briser les oppositions et à supprimer les contradictions qui sont à l'origine d'échecs enregistrés pour les femmes en matière de développement. La DDA propose ici des "lignes d'action" pour la centrale, le terrain et les régisseurs. Dans une première phase (1989-1993), la promotion des femmes - 
d'alors - était dotée d'un poste de travail à 50\%. En 1994, la capacité destinée à la deuxième phase a été accrue pour atteindre $170 \%$.

\section{Afrique du Sud}

Le déroulement du vaste processus de démocratisation et de développement en Afrique du Sud joue à n'en pas douter un rôle crucial pour l'avenir de toute la région. Au cours de longues décennies où l'apartheid était en vigueur, la Suisse a refusé de prendre des sanctions économiques contre l'Afrique du Sud. Pour encourager un changement politique dans le pays, la Suisse a commencé, dans le cadre de la coopération au développement, à soutenir des organisations privées en Afrique du Sud, notamment dans les domaines de la formation, des droits de l'homme et du dialogue politique (50 millions de francs au total entre 1982 et 1993). Au cours des cinq années à venir, elle allouera 80 autres millions de francs à l'Etat et aux organisations non gouvernementales pour les soutenir dans leurs programmes de développement et de reconstruction nationale. C'est du moins la déclaration d'intention faite par le Conseiller fédéral Flavio Cotti à l'occasion de sa visite en Afrique du Sud en septembre 1994.

\section{Palestine}

Le processus de paix engagé au Proche-Orient permet désormais d'intensifier l'aide à la Palestine. La DDA a ouvert un bureau de liaison à Jéricho, qui a pour tâche de développer les relations avec divers partenaires locaux - les autorités palestiniennes des territoires autonomes et des organisations privées. La reconstruction de la Palestine est financée par un crédit-cadre de 60 millions de francs sur cinq ans que le Conseil fédéral a approuvé en septembre 1993.

\section{Rwanda}

La coopération au développement connaît aussi des échecs. La Suisse a aussi été surprise par le conflit qui a éclaté en avril 1994 et a suspendu sa coopération avec le Rwanda. L'aide de 29,4 millions de francs qui lui était allouée en 1993 plaçait le Rwanda en tête de liste des pays africains bénéficiant des mesures bilatérales de la coopération suisse au développement. En 1994, la Suisse a principalement fourni une aide humanitaire au pays. Cette année-là, les dépenses de l'aide humanitaire et du Corps suisse en cas de catastrophe ont atteint quelque 27 millions de francs. Une décision concernant la poursuite du programme au Rwanda et dans la région sera prise lorsque la situation se sera normalisée.

\section{Comité d'aide au développement (CAD)}

La Suisse compte parmi les 22 membres (21 pays industrialisés et la Commission de l'UE) du Comité d'aide au développement de l'OCDE (CAD). Le CAD 
joue un rôle important en tant qu'organe de coordination de l'aide bilatérale. C'est en son sein que les divers pays donateurs élaborent des standards unifiés en matière de politique de développement. Le CAD a par exemple fait office de pionnier pour définir les principes de la "bonne gestion gouvernementale" et la cohérence de la coopération au développement, le développement participatif, les conditions de l'aide financière ou l'utilisation de crédits mixtes, la saisie statistique et la comptabilisation des dépenses d'APD. (Voir à ce propos l'article de Thomas Greminger sur l'aide publique au développement de la Suisse dans le contexte international, dans la deuxième partie du présent annuaire.) La coordination de l'aide mérite encore d'être améliorée. Pour y parvenir, il convient d'identifier, de discuter et si possible de résoudre les conflits d'intérêts. Sans des informations complètes, ce processus demeurera lettre morte. Les pays membres fournissent chaque année un rapport sur leur APD sous la forme d'un mémorandum et tous les trois ans deux pays examinent I'APD d'un autre membre. L'aide de la Suisse a été examinée pour la dernière fois en 1993. La qualité de cette aide a été jugée bonne, mais son volume laisse à désirer. Les autres pays membres du CAD ont prié la Suisse de fournir tous les efforts nécessaires pour que son objectif à moyen terme de porter ses dépenses d'APD à $0,40 \%$ du PNB puisse être atteint avant l'an 2000 déjà (cf. Annuaire Suisse-Tiers Monde 1994).

Des groupes de travail spéciaux abordent des thèmes spécifiques de l'APD (aide financière, statistique, évaluation, participation des femmes, environnement, développement participatif et bonne gestion gouvernementale).

\section{Aide bilatérale / Aide multilatérale}

L'aide bilatérale semble bénéficier d'un large appui auprès de la population et des oeuvres d'entraide, tandis que l'aide multilatérale est considérée d'un oeil plus critique. L'aide bilatérale de la Confédération représente en moyenne $75 \%$ et l'aide multilatérale $25 \%$ de l'aide totale, ces chiffres ne comprenant pas les cofinancements réalisés avec la Banque mondiale. En 1993, ces proportions se sont situées à 77 et $23 \%$. A l'avenir, le versement des contributions aux institutions de Bretton-Woods devrait accroître la part de l'aide multilatérale. Selon le Conseil fédéral cet accroissement sera dû aussi à "l'évolution des besoins dans les pays en développement et aux instruments dont dispose la coopération au développement internationale pour y faire face." (Message 94.029, p. 65).

Selon les estimations, la part de l'aide multilatérale devrait se stabiliser à $32 \%$ environ. Cette tendance déplaît aux oeuvres d'entraide qui craignent que la réduction des moyens mis à disposition de l'APD étant de mise - les économies soient réalisées plutôt aux dépens de l'aide bilatérale que sur le compte des engagements multilatéraux.

\section{Sondage sur l'APD}

Tous les cinq ans, la DDA et la Communauté de travail des oeuvres d'entraide 
commandent un sondage afin de connaître l'opinion des citoyens suisses sur la politique de développement. Par rapport à 1984 et à 1989, c'est un sentiment d'impuissance qui règne face aux problèmes mondiaux. Ces dernières années ayant été marquées par la récession, l'accroissement du déficit de l'Etat, l'intégration européenne et l'isolement de la Suisse, la coopération au développement perd de son intérêt auprès de la population. Le climat est donc au désintérêt et les donateurs s'essoufflent.

Dans le même temps, plus de gens sont d'avis que des actions personnelles concrètes peuvent contribuer à résoudre les problèmes globaux. La préservation de l'environnement est aujourd'hui l'un des motifs plaidant pour le maintien de l'aide au développement, tandis que la solidarité en était le principal motif il y a quatre ans encore. Le règlement du problème des réfugiés, la promotion de la paix et l'amélioration des conditions de vie des populations du Sud constituent toutefois des motifs importants. Le sondage d'opinion a été menée auprès de 1257 personnes. Voici les résultats obtenus pour quelques questions précises: $79 \%$ des personnes interrogées se sont prononcées en faveur d'une interdiction d'exporter des déchets toxiques dans les pays du Sud, $73 \%$ sont pour un accroissement des importations de sucre en provenance de pays en développement, $71 \%$ souhaitent que l'on interdise les exportations d'armes vers le Tiers Monde et $69 \%$ voudraient que l'entraide judiciaire soit accélérée pour permettre de restituer les capitaux en fuite placés dans des banques suisses.

\subsection{Dépenses de l'aide publique au développement en 1993 (3)}

Les dépenses de la Confédération, des cantons et des communes constituent ensemble l'aide publique au développement. En 1993, ces dépenses ont atteint un total de 1222 millions de francs, dont 1197 provenaient de la Confédération et 25 millions des cantons et des communes. En 1992, les dépenses d'APD s'étaient montées à 1358 millions de francs. Le recul enregistré en 1993 par rapport à l'année précédente s'explique par les dépenses extraordinaires consenties en 1992 pour financer l'adhésion de la Suisse au groupe de la Banque mondiale. L'aide fournie par les cantons et les communes a également diminué en 1993: elle est passée de 28 millions de francs en 1992 à 25 millions en 1993. (Voir tableau № 18)

Selon la méthode de calcul suisse, l'aide publique au développement a atteint en 1993 0,34\% du produit national brut (PNB). La Suisse est donc encore loin de l'objectif de $0,40 \%$ du PNB qu'elle continue pourtant de viser. Dans le plan de législature 1991-1995, le Conseil fédéral avait déclaré que le volume de l'aide publique au développement devait dans la mesure du possible atteindre $0,40 \%$ du produit national brut jusqu'à la fin du siècle. Toutefois, le nouveau plan financier établi pour les années 1996 à 1998, commence par plafonner l'aide à $0,34 \%$ du PNB. 
Tableau $\mathrm{N}^{\circ} 18$

Aperçu des flux de capitaux suisses vers les pays en développement

\begin{tabular}{|c|c|c|}
\hline & \multicolumn{2}{|c|}{$\begin{array}{lr}1992 & 1993 \\
\text { (millions de francs) }\end{array}$} \\
\hline $\begin{array}{l}\text { Coopération au développement et } \\
\text { aide humanitaire } \\
\text { - Coopération technique } \\
\text { - } \quad \text { Aide financière } \\
\text { - Mesures économiques et commerciales } \\
\text { - } \quad \text { Aide humanitaire } \\
\text { - } \quad \text { Aide alimentaire } \\
\text { - Non classé } \\
\text { - Remboursement de prêts }\end{array}$ & $\begin{array}{r}1 ' 385 \\
501 \\
386 \\
208 \\
170 \\
81 \\
45 \\
-6\end{array}$ & $\begin{array}{r}1221 \\
513 \\
228 \\
181 \\
171 \\
71 \\
63 \\
-6\end{array}$ \\
\hline Prestations privées des oeuvres d'entraide & 236 & 213 \\
\hline $\begin{array}{l}\text { Capitaux privés' } \\
\text { - } \quad \text { Investissements directs } \\
\text { - } \quad \text { Emprunts de capitaux d'institutions } \\
\text { et de pays en développement } \\
\text { - Crédits nets des banques }\end{array}$ & $\begin{array}{r}2 ' 745,7 \\
1^{\prime} 170,0 \\
766,7 \\
809,0\end{array}$ & $\begin{array}{r}6 ' 524,8 \\
1^{\prime} 618,0 \\
617,8 \\
4^{\prime} 289,0\end{array}$ \\
\hline $\begin{array}{l}\text { 1) Chiffres provisoires. } \\
\text { Source: DDAVOFAEE, Rapport annuel 1993; Mém }\end{array}$ & 3 de & $C A D$ \\
\hline
\end{tabular}

\section{L'APD: des mandats pour l'économie suisse}

Seule une partie modeste de l'aide suisse est accordée sous forme d'aide liée; c'est notamment le cas des crédits mixtes et de certaines parties de l'aide alimentaire, ce qui fait de la Suisse un modèle du genre face à d'autres pays de I'OCDE (cf. l'article de Thomas Greminger sur l'aide suisse au développement dans le contexte international). Les effets économiques de l'aide au développement n'en sont pas moins intéressants pour la Suisse. En 1992, les dépenses d'APD ont atteint 1,3 milliard de francs, tandis que l'APD internationale rapportait plus de 1,5 milliard de francs à des Suisses (expertises, conseils) et à des entreprises suisses. (Ces données sont détaillées dans la partie statistique du présent annuaire.) II est donc vrai que l'aide au développement se révèle rentable pour la Suisse, mais il convient de relativiser en précisant que la présence de la Suisse sur le "marché international" des mandats de coopération au développement n'a aucun lien avec le volume concret de son APD. C'est la capacité concurrentielle d'une entreprise qui fait la différence. De sorte que I'APD se révèle en général rentable pour des entreprises du pays donateur qui sont concurrentielles sur le plan international. 


\subsection{Aide bilatérale}

L'aide bilatérale a atteint 937 millions de francs ou $77 \%$ des dépenses totales d'APD. Elle a servi à financer 750 projets et programmes dans 80 pays, dont 18 ont bénéficié d'un soutien tout particulier car ils sont les principaux bénéficiaires de l'aide suisse. Ces 18 pays de concentration de l'aide suisse sont énumérés dans le tableau 19. La nécessité de mieux cibler l'aide réduira encore le nombre des pays dits de concentration. Le Viêt-nam viendra pourtant s'ajouter à la liste dès 1995, mais trois pays en seront exclus: Indonésie (cf. dossier); le Kenya (car l'importante corruption du gouvernement rend la coopération difficile); le Cap-Vert où la présence suisse sera réduite et stabilisée à un niveau plus faible, notamment en raison du grand engagement de l'Autriche dans ce pays. Au sein même des principaux pays bénéficiaires, l'aide se concentrera à long terme sur quelques domaines choisis; la coopération se limitera alors à trois ou quatre secteurs.

Les instruments mis en oeuvre par la DDA comprennent la coopération technique et l'aide financière, l'aide humanitaire et l'aide alimentaire. Ils représentent environ $75 \%$ de toutes les dépenses d'APD de la Confédération. Les mesures de politique économique et commerciale, quant à elles, relèvent de I'OFAEE et absorbent quelque $15 \%$ des dépenses. Le reste est réparti entre le Département de l'intérieur (formation et science) et le Département des finances. A l'occasion d'un séminaire organisé par les Archives fédérales et la DDA, les participants ont plaidé pour la mise en place d'une politique de développement sensée et cohérente. Dans cet objectif, une réforme de l'administration sera nécessaire en vue de confier la compétence globale en matière d'APD à un seul département (4).

La loi fédérale de 1976 prescrit que la coopération suisse au développement doit venir en aide aux pays les plus pauvres et aux populations les plus défavorisées. Étant donné que la majorité des pays les plus pauvres se trouvent en Afrique, au Sud du Sahara, c'est ce continent qui reçoit la majeure partie de l'aide suisse au développement (302 millions de francs). Viennent ensuite l'Asie (225 millions de francs) et l'Amérique latine ( 87 millions). (Voir à ce sujet les données détaillées dans la partie statistique du présent annuaire.)

Le Message concernant la continuation de la coopération technique et de l'aide financière en faveur des pays en développement, publié au cours de la période considérée, fait l'objet d'un commentaire ci-après.

\subsection{Nouveau crédit de programme pour la coopération technique et l'aide financière}

Les mesures de la coopération au développement sont financées par des crédits de programme que le Parlement approuve toujours pour quatre ans. L'Annuaire 1994 passe en revue tous les crédits de programme en vigueur (p. 136). Pour les années 1995 à 1999, le Conseil fédéral a proposé un nouveau crédit de programme de 3900 millions de francs afin de poursuivre la coopération 
Tableau $\mathrm{N}^{\circ} 19$

Les pays de concentration de la coopération bilatérale

La politique de concentration renforcée entraîne l'évolution suivante:

\begin{tabular}{|c|c|c|}
\hline Situation en 1992 & Situation en 1994 & Perspectives pour 1995-98 \\
\hline $\begin{array}{l}\text { Afrique occidentale } \\
\text { Bénin } \\
\text { Burkina Faso } \\
\text { Cap Vert/Guinée-Bissau } \\
\text { Ghana } \\
\text { Mali } \\
\text { Niger } \\
\text { Tchad }\end{array}$ & $\begin{array}{l}\text { Bénin } \\
\text { Burkina Faso } \\
\text { Cap Vert/Guinée-Bissau } \\
1993 \\
\text { Mali } \\
\text { Niger } \\
\text { Tchad }\end{array}$ & $\begin{array}{l}\text { Maintien } \\
\text { Maintien } \\
1995 \\
- \\
\text { Maintien } \\
\text { Maintien } \\
\text { Maintien }\end{array}$ \\
\hline $\begin{array}{l}\text { Afrique orientale } \\
\text { Kenya } \\
\text { Madagascar } \\
\text { Mozambique } \\
\text { Rwanda }{ }^{1} \\
\text { Tanzanie }\end{array}$ & $\begin{array}{l}1993 \\
\text { Madagascar } \\
\text { Mozambique } \\
\text { Rwanda } \\
\text { Tanzanie }\end{array}$ & $\begin{array}{l}\text { Maintien } \\
\text { Maintien } \\
\text { Maintien } \\
\text { Maintien }\end{array}$ \\
\hline $\begin{array}{l}\text { Asie I } \\
\text { Bangladesh } \\
\text { Inde } \\
\text { Pakistan }\end{array}$ & $\begin{array}{l}\text { Bangladesh } \\
\text { Inde } \\
\text { Pakistan }\end{array}$ & $\begin{array}{l}\text { Maintien } \\
\text { Maintien } \\
\text { Maintien }\end{array}$ \\
\hline $\begin{array}{l}\text { Asie } \|^{2} \\
\text { Indonésie } \\
\text { Népal } \\
-\end{array}$ & $\begin{array}{l}\text { Indonésie } \\
\text { Népal } \\
-\end{array}$ & $\begin{array}{l}1997 \\
\text { Maintien } \\
\text { Le Vietnam à la place de } \\
\text { l' Indonésie }\end{array}$ \\
\hline $\begin{array}{l}\text { Amérique latine }{ }^{3} \\
\text { Bolivie } \\
\text { Honduras/Nicaragua }{ }^{4} \\
\text { Pérou }\end{array}$ & $\begin{array}{l}\text { Bolivie } \\
\text { Nicaragua/Honduras } \\
\text { Pérou }\end{array}$ & $\begin{array}{l}\text { Maintien } \\
\text { Nicaragua/Amérique centr. } \\
\text { Maintien }\end{array}$ \\
\hline
\end{tabular}

\section{Total des pays de concentration de la coopération bilatérale}

1) La coopération a été suspendue après les événements intervenus en avril 1994. La question de savoir si le Rwanda pourra ultérieurement redevenir un pays de concentration devra être soumise à une réappréciation.

2) Le Bhoutan est un pays de concentration d'Helvetas qui est soutenue par la DDA. En 1995, le bureau de coordination de Bangkok sera fermé.

3) L'Equateur est un pays de seconde priorité, mais il dispose d'un bureau de coordination.

4) Le centre de gravité du programme d'Amérique centrale a été déplacé du Honduras au Nicaragua, avec un réseau régional pour l'Amérique latine.

Anciens pays de concentration (avant 1990): la Tunisie, le Paraguay et le Cameroun.

Source: Message concernant la continuation de la coopération technique et de l'aide financière en faveur des pays en développement, Berne, 1994. 
technique et l'aide financière de la Confédération. Le Parlement a approuvé le crédit de programme d'un montant de 3800 millions de francs. Ce nouveau crédit vient remplacer l'ancien crédit de programme de 3300 millions de francs qui a été épuisé à la fin de 1994. Le message concernant ce crédit - présenté au Parlement en même temps que les "Lignes directrices Nord-Sud" - concentre son analyse sur les efforts nécessaires pour encourager un développement durable.

Avant de procéder à l'analyse économique et politique du "Tiers Monde", une précision s'impose. Le Message souligne que la répartition des pays dans "trois mondes" a perdu de son sens après la disparition du "Deuxième Monde". Le "Tiers Monde" n'est pas - et encore moins que par le passé - une entité politique homogène. Les grands contrastes qui séparent par exemple les pays nouvellement industrialisés d'Asie et les pays africains au Sud du Sahara font plutôt apparaître des conflits d'intérêts entre différents pays dits du Sud. La compétition qui oppose les pays de l'Est et les pays du Sud dans la course aux capitaux et au rôle de partenaire commercial risque de marginaliser encore plus les pays les plus pauvres. C'est à cette tendance que souhaite s'opposer l'APD suisse.

Tableau $\mathrm{N}^{\circ} 20$

Ventilation par institutions bilatérales et multilatérales de la coopération au développement: Engagements 1995-1998

\begin{tabular}{|l|r|}
\hline & Mio FS \\
\hline I. Programmes bilatéraux & $2 ' 500$ \\
a) Asie & 725 \\
b) Afrique & 845 \\
c) Amérique latine & 300 \\
d) Programmes sectoriels généraux & 225 \\
e) Contributions de programmes ONG & 225 \\
f) Autres mesures bilatérales & 180 \\
II. Contributions multilatérales & \\
a) Organisations des N.U. et autres organisations & $1^{\prime} 400$ \\
b) Fonds régionaux de développement & 500 \\
c) AID & 375 \\
Total & 525 \\
\hline
\end{tabular}

Source: Message concernant la continuation de la coopération technique et de l'aide financière en faveur des pays en développement, Berne, 1994. 
En raison de leur volume, la coopération technique (513 millions de francs en 1993) et l'aide financière (228 millions de francs) sont les principaux instruments de l'APD suisse (60\% des dépenses totales en 1993), qui s'appliquent aussi bien sur le plan bilatéral que multilatéral. Le crédit de programme de 3300 millions de francs était complètement épuisé à la fin de 1994 et il sera remplacé par le nouveau crédit de 3800 millions de francs. A l'origine, le DFAE avait prévu un montant de 4150 millions de francs pour les quatre années suivantes. Vu les problèmes budgétaires de la Confédération et du "climat politique", le Conseil fédéral a réduit sa demande de crédit de programme à 3900 millions de francs dans la proposition qu'il a présentée au Parlement. Ce dernier, a accepté le crédit de programme mais a abaissé sont montant total à 3800 millions de francs.

Selon le Message, un tiers environ du crédit (1400 millions de francs) financera l'aide multilatérale et deux tiers (2500 millions de francs) l'aide bilatérale. Le tableau 20 indique les engagements qui découlent du nouveau crédit de programme pour les années 1995 à 1998.

\section{Développement durable}

"Le développement durable implique un processus qui part d'un monde différencié et en partie contradictoire et qui cherche à tenir compte des besoins des générations actuelles, sans prétériter cependant les chances des générations futures. ... Un développement durable implique une interaction dynamique et équilibrée entre tous les facteurs humains et naturels. II s'agit d'un but suprême valable pour toutes les sociétés. Pour la mise en oeuvre d'une stratégie de développement durable, les cahiers des charges des pays du Nord et du Sud sont certes différents, mais il s'agit d'harmoniser partout les divers secteurs de l'action humaine pour la faire converger vers la durabilité. ... Les plus grands dangers procédant d'une exploitation inconsidérée des ressources (désertification, changements climatiques, élévation possible du niveau de la mer) affecteraient beaucoup plus durement les pays en développement ... En outre, une généralisation du niveau de vie occidental, avec son degré actuel d'utilisation de ressources et d'énergie, n'est plus concevable. Il s'ensuit un grave dilemme que le Nord et le Sud se doivent de résoudre ensemble."

(Citation tirée du Message concernant la coopération technique et l'aide financière en faveur des pays en développement, p. 33-35.) 


\subsection{Mesures de politique économique et commerciale}

Les principaux instruments traditionnels dans ce domaine sont les crédits mixtes, l'aide à la balance des paiements et les mesures de désendettement. A l'avenir, l'OFAEE désire créer de nouveaux instruments de financement qui soient adaptés aux besoins des pays en développement.

\section{Crédits mixtes}

La Suisse participe à l'Arrangement sur les crédits à l'exportation de l'OCDE. Celui-ci a fixé des règles concernant l'octroi de crédits à l'exportation et d'aide liée ("directives de Helsinki"), qui sont opérationnelles depuis 1993. L'Arrangement a pour objectif de réduire les distorsions commerciales engendrées par les crédits d'aide liée et de mieux orienter l'aide sur les besoins du pays partenaire. Les pays de l'OCDE participant à l'Arrangement sur les crédits à l'exportation sont pour la plupart d'avis que les crédits d'aide liée ne sont plus de mise pour les projets industriels $(40 \%$ des financements mixtes octroyés jusqu'ici) et pour les projets d'infrastructure (notamment énergie et télécommunications) dans les villes ou dans les régions rurales développées. Le financement de tels projets doit en effet pouvoir être assuré par le secteur privé.

L'application de l'Arrangement de l'OCDE restreint les possibilités d'octroi de financements mixtes pour la Suisse, comme l'écrit l'Office fédéral des affaires économiques extérieures, responsable des mesures de politique économique et commerciale, dans le Rapport sur la politique économique extérieure 1993. Le nombre des opérations de financement mixte de la Suisse tend donc à diminuer, mais les paiements interviennent plus rapidement. "Les engagements pris par la Confédération dans le cadre des financements mixtes étant juridiquement contraignants, nous avons dû, pour faire face à ces décaissements, recourir une nouvelle fois à des compensations internes au détriment des autres mesures de politique économique et commerciale au titre de la coopération au développement. ..." (Rapport sur la politique économique extérieure 1993, p. 96). La Suisse s'est prononcée en faveur de l'adoption de critères plus sévères concernant l'emploi de crédits mixtes et espère que les autres pays donateurs appliqueront tous les nouvelles directives.

Seuls quelques rares accords de financement mixte ont été conclus au cours de la période en revue:

En 1993, un accord de ce type a été conclu avec le Viêt-nam, pour un montant de 25 millions de francs (part de la Confédération: 50\%). L'octroi simultané d'une aide à la balance des paiements de 15 millions de francs devrait permettre des effets de synergie entre les deux instruments. Ces fonds visent à renforcer la capacité institutionnelle du secteur bancaire.

Le montant alloué par le deuxième accord de financement mixte conclu avec la Thaillande en 1984 a fait l'objet d'une rallonge de 13,85 millions de francs (part de la Confédération: 1/3) en 1993 pour "permettre de mener à terme un important projet dans le secteur de l'énergie" (Rapport sur la politique 
économique extérieure). Le montant total de ce deuxième accord de financement mixte atteint ainsi 78,43 millions de francs. Avec le premier crédit mixte de 1979 , la somme se monte à 115 millions de francs. La presse qualifie le "projet dans le secteur de l'énergie" de "grosse saloperie" du point de vue écologique (Tages-Anzeiger, 2.3.1994). II s'agit de la centrale électrique au charbon de Mae Moh qui utilise de la lignite (du charbon de qualité inférieure contenant beaucoup de soufre) et libère de grandes quantités de substances toxiques dans l'atmosphère. L'installation nécessitait par la suite l'installation de filtres pour retenir le soufre.

Le premier accord de financement mixte conclu avec le Pakistan en 1987 a également fait l'objet d'une rallonge de 20,6 millions de francs (part de la Confédération: $40 \%$ ). Ce crédit mixte, d'un total de 110,6 millions de francs (y compris la rallonge), sert à financer un projet de télécommunications en zone rurale.

\section{Aide à la balance des paiements}

En raison du nombre croissant de pays qui se voient contraints d'entamer des programmes de réformes économiques, l'aide à la balance des paiements conservera toute son importance et va même encore s'accroître à l'avenir. De tels financements sont nécessaires "jusqu'au moment où les programmes de réformes déploient leurs effets et se traduisent par une augmentation des recettes d'exportation" (Rapport sur la politique économique extérieure, p. 99). Les aides à la balance des paiements varient selon les besoins de financement des pays bénéficiaires. Elles ne peuvent guère être planifiées à long terme.

Des aides à la balance des paiements ont été accordées à quatre pays en 1993:

- L'Éthiopie s'est vue octroyer une contribution de 10 millions de francs sous forme de cofinancement avec I'AID destinée à soutenir son programme d'ajustement structurel.

- La Suisse a conclu un accord avec le Viêt-nam sur la coopération économique qui comprend une aide à la balance des paiements de 15 millions de francs, le crédit mixte cité plus haut de 25 millions de francs, ainsi qu'une contribution de 10 millions de francs destinée à l'épurement des arriérés du Viêt-nam envers les institutions multilatérales de financement.

- L'aide d'un million de francs octroyée à Madagascar devrait contribuer à la restructuration du secteur bancaire dans ce pays.

- En Ouganda, une aide financière de 10 millions de francs sert à financer des importations prioritaires et la remise en état de stations de distribution de courant électrique.

\section{Mesures de désendettement}

Les mesures de désendettement constituent également un instrument important de la politique économique et commerciale. Elles sont abordées au chapitre 2 du présent annuaire. 


\section{Nouveaux instruments de financement}

Dans le Rapport sur la politique économique extérieure 1993, l'OFAEE annonce à nouveau (comme dans le rapport précédent) la création de nouveaux instruments de financement qui devraient être mieux adaptés à l'évolution des besoins dans les pays en développement. Selon l'Office, l'économie privée suisse participe à l'élaboration de ces nouveaux instruments. Ces instruments ont pour objectif pratique de soutenir les investissements privés et visent, en général, à soutenir le processus de libéralisation dans les pays en développement. "Ces nouveaux instruments devraient profiter avant tout aux pays en développement dans lesquels les entreprises privées tardent à réagir en dépit de l'instauration de conditions-cadres satisfaisantes." (op. cit. p. 97). Des projets pilotes faisant appel au nouveaux instruments de financement sont en préparation. L'OFAEE cite à titre d'exemple des actions de soutien à un fonds régional de financement d'entreprises de leasing en Afrique (EDFUND) et à un fonds de promotion de petites entreprises en Amérique latine (PROFUND). Les nouveaux instruments devraient devenir pleinement opérationnels dans le cadre du cinquième crédit de programme (dès 1995).

\subsection{Aide humanitaire}

L'aide humanitaire de la Confédération est un élément important de la politique extérieure de la Suisse et contribue à répondre aux besoins humanitaires croissants de par le monde. Environ $20 \%$ de l'ensemble de l'aide publique de la Confédération sont consacrés à des mesures d'aide humanitaire - celle-ci comprend également l'aide alimentaire. Ce domaine relève de la compétence de la Division aide humanitaire et Corps suisse en cas de catastrophes (ASC) de la DDA. La direction en incombe au délégué à l'aide humanitaire.

En 1994, le directeur de la DDA, Walter Fust, a adopté la "Stratégie de l'aide humanitaire de la Confédération pour la seconde moitié des années nonante". Cette stratégie fixe des priorités pour les activités sur place en faveur des victimes de catastrophes naturelles, de celles dites de civilisation et de celles provoquées par des conflits armés. La Stratégie de l'aide humanitaire présentée pour la première fois sous cette forme - se fonde sur les "Lignes directrices Nord-Sud" pour les années 90 et formule les particularités de l'aide humanitaire. Celle-ci doit être universelle, c'est-à-dire que tous les pays et tous les groupes de population doivent pouvoir en bénéficier. L'existence d'une situation d'urgence est le principal critère d'octroi de l'aide. L'aide est neutre et impartiale; elle n'est pas liée à des conditions politiques. L'aide suisse est octroyée selon le principe du respect de la dignité humaine, sans distinction de race, de sexe, de langue, de religion, d'appartenance politique ou sociale.

L'aide humanitaire comprend les projets de la DDA, les actions du Corps suisse d'aide en cas de catastrophes et le soutien accordé dans le domaine de la coopération humanitaire à des programmes d'oeuvres d'entraide suisses et 
d'organisations internationales, y compris le ClCR. Ce soutien est fourni sous forme d'aide en espèces ou en nature (denrées alimentaires). Dans le courant d'une année, quelque 80 membres de I'ASC sont engagés dans des actions d'aide d'urgence, d'aide de survie, de reconstruction et de prévention à plus long terme.

En 1993, les dépenses de l'aide humanitaire se sont montées à 237 millions de francs. Ce montant comprend les 55 millions de francs versés à titre de contribution au budget du siège du CICR et un million de francs alloué au budget administratif de l'Organisation internationale pour les migrations (OIM). Ces deux contributions reposent sur deux Arrêtés fédéraux distincts et leur financement est assuré par d'autres sources. En 1993, environ 181 millions de francs ont ainsi été prélevés sur le crédit de programme pour l'aide humanitaire. Les aides les plus importantes ont été accordées à l'ex-Yougoslavie et au Rwanda.

\section{Notes}

1. Cette année, la structure du chapitre consacré à la coopération au développement a été modifiée. Les commentaires sont essentiellement consacrés à la politique de la coopération au développement et aux nouveaux éléments apparus pendant la période considérée (1993 à septembre 1994), en particulier au nouveau crédit de programme pour la coopération technique et l'aide financière. Dans la partie "Analyses et positions", l'accent est également mis sur l'un des aspects de la coopération au développement, notamment la cohérence des relations de la Suisse avec I'Indonésie. Ce dossier explique et reproduit également les "Lignes directrices Nord-Sud". Un autre article (de Thomas Greminger) analyse l'aide publique au développement de la Suisse dans le contexte international. La partie "Statistiques" (III. Aide publique au développement) présente et commente en détail les dépenses consacrées à l'aide au développement selon leur provenance, leur destination, les régions et les secteurs. Afin d'éviter les répétitions, les tableaux de la partie statistique ne sont plus reproduits dans la partie "Revue".

2. Dans le dossier, les auteurs des divers organes concernés (administration, économie privée, oeuvres d'entraide, syndicats et universités) expliquent les problèmes, les mécanismes et les instruments qui interviennent dans la mise en place d'une cohérence optimale dans la coopération au développement entre la Suisse et l'Indonésie.

3. Les chiffres sont tirés du Rapport annuel 1993 de la DDA et de I'OFAEE. Pour classei les pays dans les différents groupes, la DDA et l'OFAE se fondent sur la définition de l'OCDE. Celle-ci classe l'Albanie, la Turquie, les pays de l'ex-Yougoslavie, ainsi que l'Arménie, la Géorgie, l'Azerbaïdjan, le Kirghizistan, l'Ouzbékistan, le Turkménistan, le Kazakhstan et le Tadjikistan parmi les pays en développement.

4. Le séminaire se fondait sur des études complètes concernant la politique suisse de développement qui ont été publiées dans le volume 19 de "Studien und Quellen" des Archives fédérales: Von der Entwicklungshilfe zur Entwicklungspolitik. Voir à ce propos également l'article de Beatrix Mesmer et de Peter Hug dans la deuxième partie du présent annuaire.

\section{Sources}

Rapport sur la politique extérieure de la Suisse dans les années 90, Berne, novembre 1993 (93.098)

Rapport du Conseil fédéral sur les relations Nord-Sud de la Suisse dans les années 90 ("Lignes directrices Nord-Sud), Berne, mars 1994 (94.003) 
Rapport sur la politique économique extérieure 93/1+2, Berne, janvier 1994 (94.007)

Message concernant la continuation de la coopération technique et de l'aide financière en faveur des pays en développement, Berne, avril 1994 (94.029)

DDAOFAEE, Rapport annuel 1993, Berne, 1994

OCDE, CAD - Rapport 1993 (Efforts et politiques des Membres du Comité d'Aide au Développement, L'aide en transition), Paris, mars 1994

Communauté de travail Swissaid / Action de carême / Pain pour le prochain / Helvetas / Caritas, Prises de position de la politique de développement 1993

Ib. id, Die Nord-Sud-Beziehungen in der offentlichen Meinung, annexe à "aktuell" nº 4/1994

DDA, Service de presse $(1993,1994)$

DDA, Conduire un développement équilibré hommes-femmes, Berne, 1993

DDA, La coopération au développement et l'aide humanitaire de la Suisse, 1993/1994, Berne, 1994 (annexe au Rapport annuel 1993)

DDA/OFAEE, Mémorandum de la Suisse au Comité d'aide au développement de l'OCDE 1992, Berne, juillet 1993; et 1993, Berne, juillet-aoat 1994

Peter Hug et Beatrix Mesmer (éd.), von der Entwicklungshilfe zur Entwicklungspolitik, Archives tédérales, Berne, 1993

DDA, Division Aide humanitaire et Corps suisse d'aide en cas de catastrophe (ASC), Stratégie de l'aide humanitaire de la Confédération pour la seconde moitié des années nonante, Berne, février 1994

Tages-Anzeiger, 21.9.1993 (60 millions d'aide à la reconstruction pour la Palestine)

Tages-Anzeiger, 2.3.1994 (Crédit mixte suisse pour la Thaillande)

NZZ, 15.9.1994 (80 millions de francs pour l'Afrique du Sud)

Tages-Anzeiger, 27.9.1994 (Etat des lieux au Rwanda)

Tages-Anzeiger, 15.9.1994 (Budget 1995)

\section{Aide privée au développement}

\section{3: recul des moyens à disposition}

En 1993, les dépenses de l'aide privée au développement, provenant de dons, ont atteint 213 millions de francs, contre 236 millions de francs en 1992. A ce montant viennent s'ajouter les prestations fournies par la Confédération dans le cadre de l'APD à titre de contribution aux programmes des oeuvres d'entraide ou sous forme de mandats de régie (134 millions en 1993, 132 millions de francs en 1992). II convient de relever que les prestations de la Confédération ont pour la première fois stagné en 1993, alors qu'elles ont suivi une progression régulière au cours des années précédentes. Comme les dons privés, les dépenses des cantons et des communes pour l'aide au développement ont également diminué en 1993 (25 millions de francs en 1993, 28 millions en 1992). Les cantons et les communes ne gèrent eux-mêmes qu'une partie infime de leurs dépenses d'APD (2 millions de francs environ); la majeure partie est en effet administrée par les oeuvres d'entraide. Dans l'ensemble, l'aide au développement gérée et réalisée par des organismes privés a ainsi reculé de 396 millions de francs en 1992 à 372 millions de francs en 1993 . Ce recul ne revêt pas les mêmes proportions auprès de toutes les oeuvres d'entraide. En Suisse, plus de 400 oeuvres d'entraide travaillent dans le domaine de la coopération au développement. La partie statistique du présent annuaire présente l'aide fournie par 25 des plus grands de ces organismes. (1) 
Les moyens destinés à l'aide aux pays de l'Est dont les oeuvres d'entraide ont pu disposer en 1993 étaient également inférieurs à ceux de l'année précédente et ont atteint 15 millions de francs contre 19 en 1992 et 21 en 1991. L'aide aux pays d'Europe de l'Est et de l'ex-Union soviétique a tout d'abord été fournie sous forme d'aide humanitaire, qui devrait désormais être remplacée par la coopération technique.

Afin de coordonner leur aide à l'Est, les oeuvres d'entraide Caritas, Helvetas, Intercooperation et Swisscontact ont créé en 1993 un groupe de travail sur l'Asie centrale qui a pour tâche de préparer l'extension de leur aide à cette région. Dans ce cadre, le Kirghizistan pourrait devenir le principal pays bénéficiaire de leur aide concertée. Le Tadjikistan ne bénéficie pas de l'aide à l'Est de la Confédération, car ce pays ne respecte pas les droits de l'homme et qu'il tarde à entreprendre des réformes économiques et politiques. Caritas prépare un projet pilote d'aide humanitaire destinée au Tadjikistan destiné à venir en aide aux populations nécessiteuses de la zone ravagée par la guerre civile. (2)

La diminution des fonds à disposition devrait également inciter les oeuvres d'entraide - comme la Confédération pour l'APD - à mieux concentrer et coordonner leur aide. La Communauté de travail des oeuvres d'entraide écrit ainsi dans son introduction aux "Prises de position de la politique de développement 1993": "La lutte pour accroître le volume de la coopération au développement s'est poursuivie en 1993." La Communauté demande à la Confédération de libérer de nouveaux fonds pour financer les nouveaux domaines d'activités de I'APD. La taxe à la valeur ajoutée et l'augmentation des droits de douane sur les combustibles devraient fournir les recettes supplémentaires nécessaires.

\section{Dialogue sur la politique de développement}

Les oeuvres d'entraide sont des partenaires importants de la Confédération pour la réalisation de l'APD. La Confédération soutient les oeuvres d'entraide en subventionnant leurs propres programmes et en leur confiant l'exécution de projets en régie. Les oeuvres d'entraide jouent par ailleurs un rôle important dans le débat concernant la politique suisse de développement, rôle qui a été revalorisé ces dernières années. La Communauté de travail des cinq oeuvres d'entraide Swissaid, Action de carême, Pain pour le prochain, Helvetas et Caritas occupe une place prépondérante dans ce débat. Au cours de la période sous revue, elle a pris position sur nombre de thèmes, dont notamment le GATT, l'environnement, le désendettement, le FMI et la Banque mondiale et ainsi que sur les thèmes abordés lors des conférences internationales, comme celle sur la population (voir à ce propos les chapitres correspondants).

Dans leur prise de position relative au Rapport du Conseil fédéral sur la politique extérieure, les oeuvres d'entraide critiquent la prédominance de la question européenne qui occulte les problèmes de régions entières telles que l'Afrique. La Communauté de travail a demandé au Conseil fédéral de faire des 
relations avec l'Afrique une priorité de la politique extérieure suisse. Les oeuvres d'entraide soutiennent la Confédération dans ses efforts pour adhérer à l'ONU, une adhésion qu'elles souhaiteraient se voir réaliser le plus vite possible. Elles regrettent toutefois que le Rapport sur la politique extérieure ne prévoit aucun calendrier à ce propos.

Pour permettre aux directeurs exécutifs de la Suisse auprès du FMI et de la Banque mondiale, Daniel Kaeser et Daniel Gerber, de se faire une idée des conséquences engendrées par le processus d'ajustement structurel à tous les niveaux de la société, la Communauté de travail a organisé un voyage à travers le Ghana auquel d'autres représentants de l'administration ont également pris part. Ce voyage avait pour objectif de lancer un processus d'apprentissage commun sur la base de la politique d'ajustement structurel. Le dialogue politique entre les oeuvres d'entraide privées et l'administration et le Parlement est institutionnalisé dans la mesure où des représentants de ces organismes siègent dans diverses commissions fédérales. Pour ce qui est de la politique de développement, la Communauté des oeuvres d'entraide collabore aussi avec d'autres organisations non gouvernementals au niveau européen (EUROSTEP), ainsi que dans le cadre de groupes de travail consacrés à des thèmes spécifiques (Banque mondiale, commerce, environnement, etc.). Dans le cadre d'EUROSTEP, ces organismes ont entrepris pour la première fois en 1993 une estimation de l'APD mondiale, c'est-à-dire du total de l'APD de tous les pays donateurs. Cette étude a été publiée sous le titre "The Reality of AID" (3).

Les oeuvres d'entraide ont salué la publication des "Lignes directrices NordSud" du Conseil fédéral. Selon elles, ce rapport appréhende avec beaucoup de justesse les problèmes et le contexte dont il faut tenir compte pour mettre en place une APD cohérente, mais omet hélas totalement de mentionner les instruments pour y parvenir. En avril 1994, au moment de la publication des "Lignes directrices Nord-Sud", les oeuvres d'entraide ont demandé à la Confédération d'accroître l'efficacité de l'aide au développement: à l'avenir, la Confédération devrait se consacrer presque exclusivement à la coordination de l'APD au sein de son administration et dans le cadre des instances internationales, et confier plus (que par le passé) l'exécution concrète des projets aux oeuvres d'entraide.

La Communauté de travail des oeuvres d'entraide accomplit une tâche essentielle de coordination au niveau de l'opinion et du travail d'information, sans prétendre pour autant représenter toute la diversité des avis que l'on retrouve parmi les 400 organisations privées. En effet, chacune de ces organisations possède sa propre motivation, son propre domaine de travail et ses propres membres (4).

\section{Oeuvres d'entraide plus petites}

Dans chaque annuaire, nous ne pouvons présenter que quelques-unes des nombreuses oeuvres d'entraide confessionnelles ou laïques. Les annuaires de 
ces dernières années ont mis l'accent sur les oeuvres d'entraide relativement grandes, car ce sont elles qui administrent la majeure partie de l'aide. Les sept oeuvres d'entraide les plus grandes gèrent en effet $45 \%$ de l'aide privée au développement.

A titre représentatif pour nombre d'autres organismes, nous présentons cette année une oeuvre d'entraide de petite taille et une organisation de la politique de développement. Les petites organisations jouissent d'une bonne réputation auprès de la population, d'une part parce que leurs structures sont simples et, d'autre part, parce qu'elles sont très compétentes puisqu'elles limitent souvent leur aide à un domaine précis.

La Fondation Vivamos mejor fait partie des petites organisations. II s'agit d'une fondation qui gère environ 20 projets dans six pays d'Amérique latine (pour un total de 410000 francs en 1993). Sa concentration est orientée sur l'Amérique latine du point de vue géographique et sur les projets mère-enfant pour ce qui est du secteur social. La fondation, créée en 1981, constate que la thèse de la "décennie perdue" se confirme dans son travail. Le "démantèlement du secteur de la santé et de l'éducation", qui est une conséquence de la crise de l'endettement et les réformes structurelles, rend le soutien aux groupes d'entraide d'autant plus nécessaire.

Le Solifonds (fonds de solidarité pour la libération sociale dans le Tiers Monde) est également une fondation, qui est "issue du point de vue que la coopération au développement et l'aide humanitaire au Tiers Monde sont insuffisantes si elles ne sont pas accompagnées d'un processus de libération sociale et politique" (citation tirée d'un dépliant du Solifonds). Le Solifonds a été fondé en 1983 par l'Union syndicale suisse, le Parti socialiste suisse et l'Oeuvre suisse d'entraide ouvrière (OSEO) et il est alimenté par diverses organisations de coopération au développement. II soutient des actions politiques limitées dans le temps mises sur pied par des organisations de base, des syndicats et des groupements de défense des droits de l'homme dans le Tiers Monde. Voici quelques exemples de projets appuyés par Solifonds: une grève chez Everite (une multinationale suisse) en Afrique du sud, une aide initiale pour un projet autonome des habitants de bidonvilles aux Philippines, soutien de la lutte salariale menée par les travailleurs de la canne à sucre au Brésil, travail d'information sur les droits des populations indiennes au Mexique. Les dépenses consacrées à des projets dans des pays en développement se montaient en 1993 à 74000 francs.

Notes

1. Les prestations fournies par plus de 200 des oeuvres d'entraide privées dans le cadre de la coopération au développement avec les pays du Sud et de l'Est sont recensées en détail dans la publication annuelle de l'IUED et de la DDA qui a pour titre: Aide suisse aux pays en développement et aux pays de l'Europe orientale. 
2. En septembre 1993, des représentants des quatre oeuvres d'entraide ont séjourné pendant un mois au Kirghizistan et analysé la possibilité de mettre sur pied des projets de coopération technique. Les impressions du voyage sont publiés sous forme de dossier dans l'édition de décembre 1993 de "mosquito".

3. The Reality of Aid - An independent Review of international Aid, 1993 et 1994. A l'avenir, ce rapport devrait paraittre chaque année à titre de complément critique au rapport annuel officiel du CAD de l'OCDE.

4. Pour avoir des informations sur les oeuvres d'entraide et leurs domaines de concentration, on peut se référer à leurs rapports annuels. Les grandes oeuvres d'entraide publient également leurs propres journaux et bulletins qui sont régulièrement envoyés à leurs membres.

\section{Sources}

Communauté de travail Swissaid / Action de carême / Pain pour le prochain / Helvetas / Caritas, Prises de position de la politique de développement 1993, Berne, 1994

lb. id. Rapport annuel 1993

Oeuvre suisse d'entraide ouvrière (OSEO), L'avenir est à tout le monde - La coopération au développement, aujourd'hui et demain, juillet 1993

Dépliant du Solifonds 1994

Tages-Anzeiger, 7.9.1993 (The Reality of Aid)

Tages-Anzeiger, 20.4.1994 (Confier le travail de terrain aux oeuvres d'entraide)

mosquito, dossier sur l'Asie centrale, décembre 1993 